\title{
Organization and Bifurcation of Planar Closed Orbits of an Atomic Electron in Crossed Fields
}

D. M. Wang

William \& Mary

John B. Delos

William \& Mary, jbdelos@wm.edu

Follow this and additional works at: https://scholarworks.wm.edu/aspubs

Part of the Physics Commons

\section{Recommended Citation}

Wang, D. M. and Delos, John B., Organization and Bifurcation of Planar Closed Orbits of an Atomic Electron in Crossed Fields (2001). Physical Review A, 63(4).

https://doi.org/10.1103/PhysRevA.63.043409

This Article is brought to you for free and open access by the Arts and Sciences at W\&M ScholarWorks. It has been accepted for inclusion in Arts \& Sciences Articles by an authorized administrator of W\&M ScholarWorks. For more information, please contact scholarworks@wm.edu. 


\title{
Organization and bifurcation of planar closed orbits of an atomic electron in crossed fields
}

\author{
D. M. Wang and J. B. Delos \\ Physics Department, College of William \& Mary, Williamsburg, Virginia 23187-8795
}

(Received 3 August 2000; published 15 March 2001)

\begin{abstract}
We describe the patterns of creation and splitting of planar closed orbits of electrons in hydrogen atoms in crossed electric and magnetic fields. These orbits lie in the plane perpendicular to the magnetic field, and they start and end at the nucleus. Using a Poincaré map to study the regular motions, we observe that the bifurcations of planar closed orbits fall into an ordered sequence as energy changes: a "tangent bifurcation'" creates one closed orbit that splits into two; subsequently, one of them becomes periodic, and splits by a "pitchfork bifurcation" into two periodic orbits and one closed orbit. Based on these calculations, we classify the closed orbits that are involved in a sequence of bifurcations in a family, and we name the family by the winding ratio of the periodic orbits in the family. To understand this ordered sequence of bifurcations, we create a simple integrable Hamiltonian as a model of the Poincare map. This model gives a simple interpretation of the sequence of the bifurcations. The model contains only general assumptions, so we expect that such sequences of bifurcations of closed orbits will be commonly found in physical systems.
\end{abstract}

DOI: 10.1103/PhysRevA.63.043409

\section{INTRODUCTION}

We study the planar orbits of an electron in a hydrogen atom in crossed electric and magnetic fields. According to closed-orbit theory, each closed orbit produces a sinusoidal oscillation in the absorption spectrum as a function of energy, and it produces a peak in the recurrence spectrum. As the energy changes, new closed orbits are created via bifurcations, so new peaks appear in the recurrence spectrum. Therefore, the patterns of bifurcations and the organization of closed orbits are of fundamental interest for understanding the spectrum. These have been studied experimentally and theoretically for Rydberg atoms in a magnetic field $[1,2]$ or in an electric field $[4,3]$ separately as well as in parallel electric and magnetic fields $[5,6]$.

In [7], Gao organized the closed orbits of a pure electric field system by their winding ratio. In this integrable system, all orbits form tori, and periodic orbits correspond to tori with rational winding numbers. Each such rational torus is filled by a family of periodic orbits with fixed action variables and varying relative phases. In the pure electric field, each orbit that is closed at the nucleus is one of these periodic orbits. Therefore, Gao showed that we can understand the closed orbits by understanding the rational tori. As the energy increases, a rational torus, together with its associated closed orbit, bifurcates out of the "downhill" orbit, evolves to an uphill orientation, and then disappears by merging with the "uphill" orbit.

The crossed-field system is completely different. Part of the phase space is filled with chaotic orbits. However, at low energies, regular orbits dominate, so we can gain insight by first focusing our attention on these. Furthermore, the measurements done by Raithel et al. [8] proved that planar closed orbits make significant contributions to the recurrences. Therefore, we restrict our attention to orbits which lie in the plane perpendicular to the magnetic field.

We find that because this system has lower symmetry than the parallel-fields system, in general closed orbits do not correspond to periodic orbits. Therefore, little of the welldeveloped theory of bifurcations of periodic orbits can be
PACS number(s): 32.80.-t, 05.45.-a, 03.65.Sq, 32.60.+i

applied. We get new patterns of creation and splitting of closed orbits. Some of these patterns are sufficiently similar to the patterns seen in bifurcations of periodic orbits that we can use the same names. We will refer to a "tangent bifurcation" and a "pitchfork bifurcation." However, it must be remembered that we are dealing here with a different phenomenon.

In this paper, we first present an "observed" sequence of bifurcations of closed orbits (observed by numerical experiment). Then we define a half-Poincaré map that we use to interpret observations. We create a simple model Hamiltonian to model the half-map and illustrate the sequence of bifurcations of closed orbits. Subsequently, we show that bifurcations of the closed orbits in the crossed-field system fall into the same sequence as that in the model Hamiltonian. Finally, we present a way to organize the planar closed orbits (where "planar" means the $x y$ plane) into families and assign each family a family name and each member of a family a given name.

"Closed" always means closed at the nucleus; typically the electron leaves the atom in one direction and returns from another, and the orbit is not periodic. However, as we will see below, some orbits are both closed and periodic.

\section{THE OBSERVED SEQUENCE OF BIFURCATIONS OF THE CLOSED ORBITS}

We have observed two typical sequences of bifurcations of closed orbits. One is shown in Fig. 1. In a region of phase space where there is no closed orbit, as the energy is decreased, abruptly a closed orbit appears. We name this one $C_{2,3}^{0}$; the names will be explained as we develop the theory. This closed orbit immediately splits into two closed orbits $\left(C_{2,3}^{-}, C_{2,3}^{+}\right)$. We call this creation of a new closed orbit and the splitting of this orbit into two closed orbits a tangent bifurcation. As the energy decreases further, each of these closed orbits changes its shape, and, in particular, $C_{2,3}^{+}$ evolves until at some energy it becomes both closed at the nucleus and periodic. We then call it $P_{2,3}$. Then two other periodic orbits $\left(P_{2,3}^{+}, P_{2,3}^{-}\right)$split out of $P_{2,3}$, while $P_{2,3}$ re- 


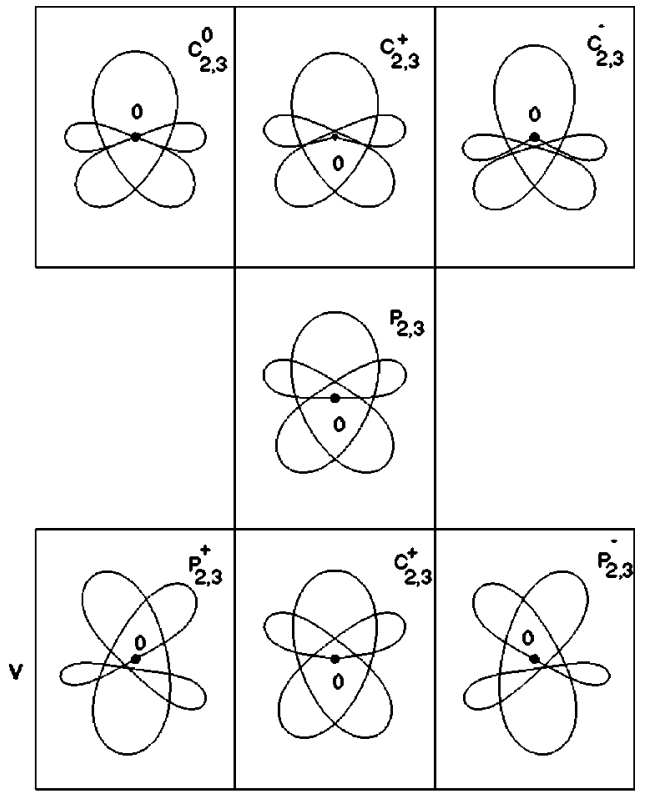

u

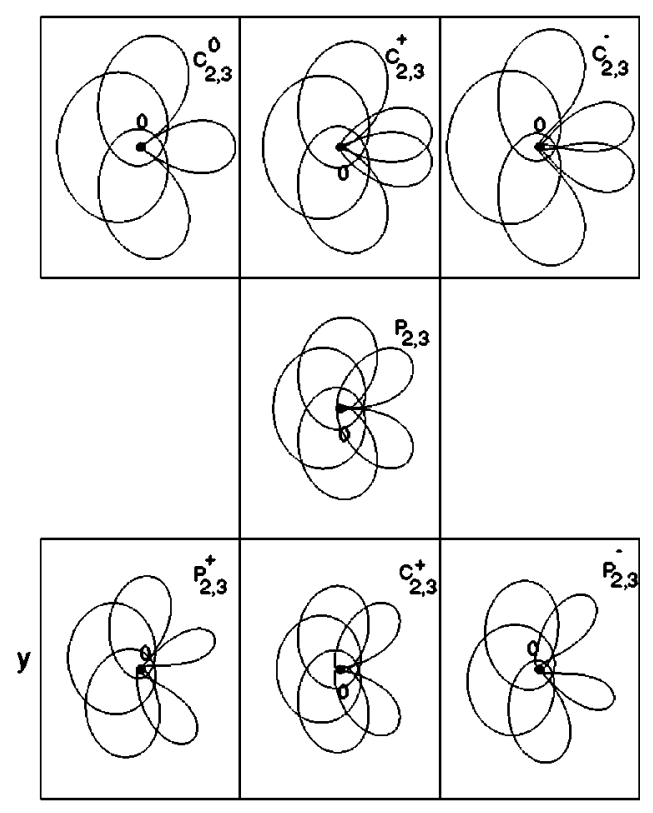

$x$

FIG. 1. An example of the normal sequence of bifurcations of closed orbits. A single closed orbit $C_{2,3}^{0}$ is created which splits into two, $C_{2,3}^{+}$and $C_{2,3}^{-}$(tangent bifurcation). One of them becomes periodic, and then splits off two periodic orbits, becoming again closed but nonperiodic (pitchfork bifurcation). The orbits are shown in $(u, v)$ space and in $(x, y)$ space. The $+x$ axis and the $\pm u$ axis are "uphill" in the electric potential. The Lorentz force pushes the electron in a counterclockwise sense in either representation.

turns to being closed but nonperiodic. We again call the closed orbit $C_{2,3}^{+}$. We call the splitting of one periodic orbit into two periodic orbits and one closed orbit a pitchfork bifurcation. Therefore, we observe that the closed orbits appear in a group of four in an orderly sequence. We call this the "normal sequence" of bifurcations of closed orbits.

A different sequence is shown in Fig. 2. A closed orbit

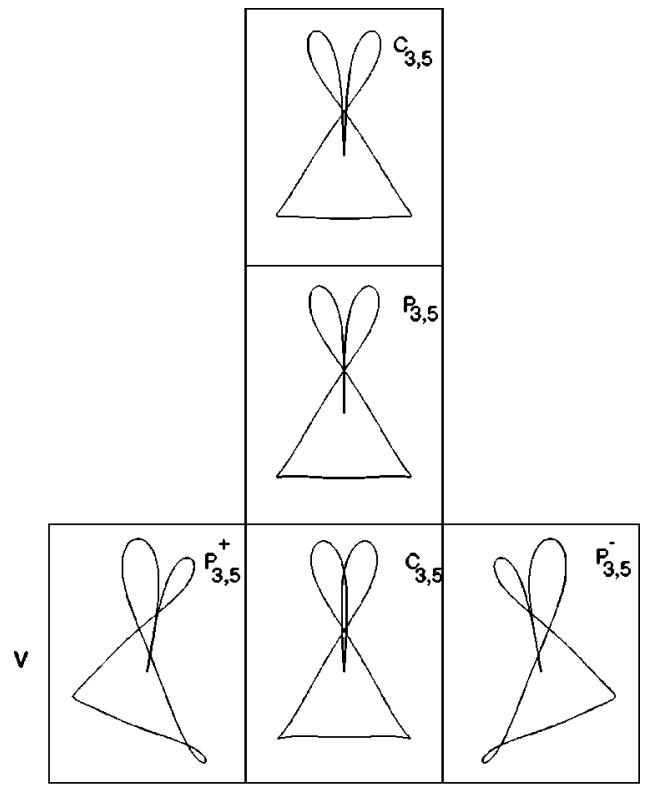

u

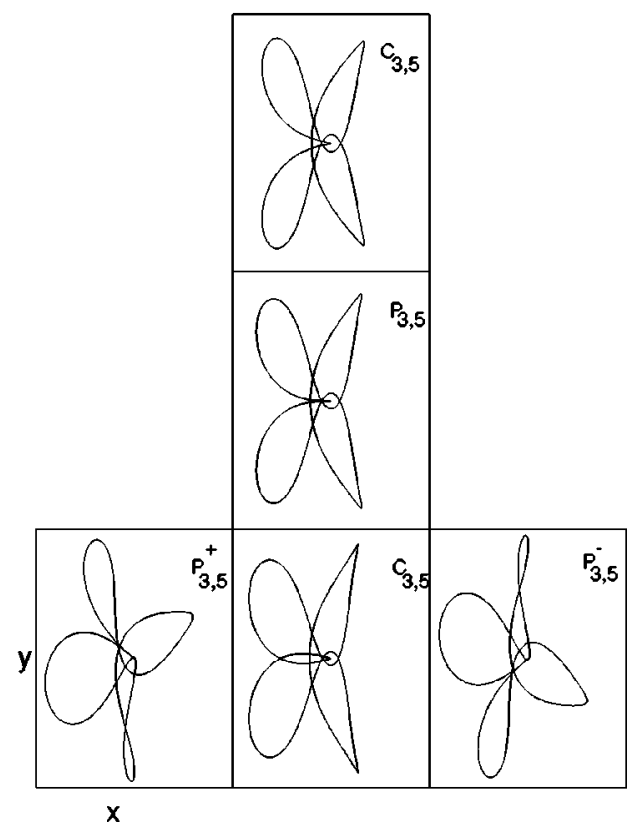

FIG. 2. An example of the truncated sequence of bifurcations. The orbit $C_{3,5}$ exists at low energies, and as the energy is raised, it undergoes a pitchfork bifurcation. Here we show half of the periodic orbits $P_{3,5}^{ \pm}$.

$C_{3,5}$ exists at very low energies. As energy increases, it evolves into one periodic orbit $P_{3,5}$ first, then becomes a nonperiodic orbit $C_{3,5}$, and two periodic orbits $\left(P_{3,5}^{+}, P_{3,5}^{-}\right)$ are created. Here, we observe that the closed orbits appear in a group of three in an orderly sequence. We call this the "truncated sequence", of bifurcations of closed orbits.

Why are such numerical observations interesting? First, from the perspective of atomic spectroscopy, it would be good to understand the orbits and their bifurcations. For example, Raithel and Walther [8] measured the recurrence spectrum of $\mathrm{Rb}$ atoms in crossed fields, and at one particular scaled energy they observed a peak associated with the orbit 
we call $C_{3,5}$. More generally, they found that recurrences are strongest near bifurcations, and indeed, for atoms in crossed fields, many recurrences are strong enough to be visible only near bifurcations.

Second, from the perspective of bifurcation theory, these observations beg for an explanation. (i) Within the oneparameter theory of bifurcations of periodic orbits, pitchfork bifurcations are nongeneric [9]. That might lead one to expect that pitchfork bifurcations of closed orbits are also nongeneric. However, we will argue in Appendix A that in integrable systems, pitchfork bifurcations of closed orbits are in fact generic. (ii) More fundamentally, bifurcation theory is usually formulated as a local theory [10]. The theorems describe what happens typically in a sufficiently small region of phase space and parameter space. What we would expect typically is tangent bifurcations. When we report that what we normally see is a tangent bifurcation followed by a pitchfork bifurcation, this observation falls outside of the local formulation of bifurcation theory: the phrase "soon followed by" means "after a finite step" in energy, not within a sufficiently small neighborhood. We need an explanation of why such generic-nongeneric bifurcations are often seen as a couple.

The purpose of this paper is to understand these sequences of creation and splitting of closed orbits. We first give more detail about the crossed-field system, then we create a simple model. This simple model has sequences of bifurcations of closed orbits that are analogous to the "normal sequence" and the "truncated sequence" of bifurcations in the crossed-field system. Most important, the model involves only general assumptions; this leads us to believe that such sequences of bifurcations of closed orbits will be commonly found in many physical systems.

\section{THE REDUCED HAMILTONIAN AND TWO FUNDAMENTAL PERIODIC ORBITS}

This section is devoted to the transformations of the reduced Hamiltonian, including rescaling and regularizing. We also discuss the role of two fundamental periodic orbits in organizing the planar orbits.

\section{A. The Hamiltonian}

\section{Reduction and regularization}

We ignore the motion of the proton [11], and we consider the motion of an electron in combined Coulomb fields plus magnetic field pointing in the $+z$ direction plus electric field pointing in the $+x$ direction, so the $+x$ direction is "uphill." The reduced two-dimensional (2D) Hamiltonian is

$$
H=\frac{1}{2}\left(\hat{P}_{\hat{x}}^{2}+\hat{P}_{\hat{y}}^{2}\right)-\frac{1}{\hat{\rho}}+F \hat{x}+\frac{B}{2} \hat{L}_{\hat{z}}+\frac{B^{2}}{8}\left(\hat{x}^{2}+\hat{y}^{2}\right)
$$

with $\hat{\rho}=\sqrt{\hat{x}^{2}+\hat{y}^{2}}$. We scale the coordinates and the parameters according to

$$
f=F B^{-4 / 3}, \quad \epsilon=E B^{-2 / 3}, \quad r=\hat{r} B^{2 / 3}, \quad P=\hat{P} B^{-1 / 3},
$$

transform to semiparabolic coordinates,

$$
\begin{gathered}
x=\frac{1}{2}\left(U^{2}-V^{2}\right), \quad P_{x}=\frac{U P_{U}-V P_{V}}{U^{2}+V^{2}}, \\
y=U V, \quad P_{y}=\frac{U P_{V}+V P_{U}}{U^{2}+V^{2}},
\end{gathered}
$$

and make one more rescaling of the variables,

$$
\begin{gathered}
U=u / \sqrt{\omega}, \quad P_{U}=p_{u} \sqrt{\omega}, \\
V=v / \sqrt{\omega}, \quad P_{V}=p_{v} \sqrt{\omega}, \\
\omega=\sqrt{-2 \epsilon}
\end{gathered}
$$

to put the Hamiltonian in the form:

$$
\begin{aligned}
\frac{2}{\omega}= & \frac{1}{2}\left(p_{u}^{2}+p_{v}^{2}\right)-\frac{1}{2}\left(u^{2}+v^{2}\right)+\frac{f}{2 \omega^{3}}\left(u^{4}-v^{4}\right) \\
& +\frac{1}{4 \omega^{2}}\left(u p_{v}-v p_{u}\right)\left(u^{2}+v^{2}\right)+\frac{1}{32 \omega^{4}}\left(u^{2}+v^{2}\right)^{3} .
\end{aligned}
$$

The coordinate transformation (2), (3), (4), and (5) maps the whole $x y$ plane to the half $(u, v)$ plane. The $\pm u$ axis corresponds to the $+x$ axis ("uphill"), and the $\pm v$ axis corresponds to the $-x$ axis ("downhill"). Therefore, we only need to consider the trajectories launched with $\dot{u}>0$. Alternatively, we may consider those launched with $\dot{v}>0$. The original system (1) has three parameters: $F, B$, and the value of $H(\mathbf{p}, \mathbf{q})=E$; reduction to Eq. (7) shows that only two parameters are significant, $(\epsilon, f)$ or $(\omega, f)$.. In this work, we will vary one parameter $(E)$.

\section{The symmetry properties of the regularized two-dimensional Hamiltonian}

The differential equations generated by the original Hamiltonian (1) are invariant under the symmetry operation, $T \sigma_{y}$, the combination of "time reversal" $T$ with reflection in $y$ :

$$
\begin{gathered}
\sigma_{y}:\left(y \rightarrow-y, P_{y} \rightarrow-P_{y}\right), \\
T:(\mathbf{P} \rightarrow-\mathbf{P}, t \rightarrow-t), \\
T \sigma_{y}:\left(x, y, P_{x}, P_{y}, t\right) \rightarrow\left(x,-y,-P_{x}, P_{y},-t\right) .
\end{gathered}
$$

The regularized equations generated by Eq. (7) are invariant under inversion and two time-reversal reflections:

$$
\begin{gathered}
\sigma_{u} \sigma_{v}:\left(u, v, p_{u}, p_{v}, t\right) \rightarrow\left(-u,-v,-p_{u},-p_{v}, t\right), \\
T \sigma_{u}:\left(u, v, p_{u}, p_{v}, t\right) \rightarrow\left(-u, v, p_{u},-p_{v},-t\right), \\
T \sigma_{v}:\left(u, v, p_{u}, p_{v}, t\right) \rightarrow\left(u,-v,-p_{u}, p_{v},-t\right) .
\end{gathered}
$$

Therefore, any orbit in $\left(u, v, p_{u}, p_{v}\right)$ space that does not have any symmetry must have three siblings that result from these three symmetry operations. Two orbits in $\left(u, v, p_{u}, p_{v}\right)$ space that are related by the inversion operation $\sigma_{u} \sigma_{v}$ are identical in $\left(x, y, P_{x}, P_{y}\right)$ space. 

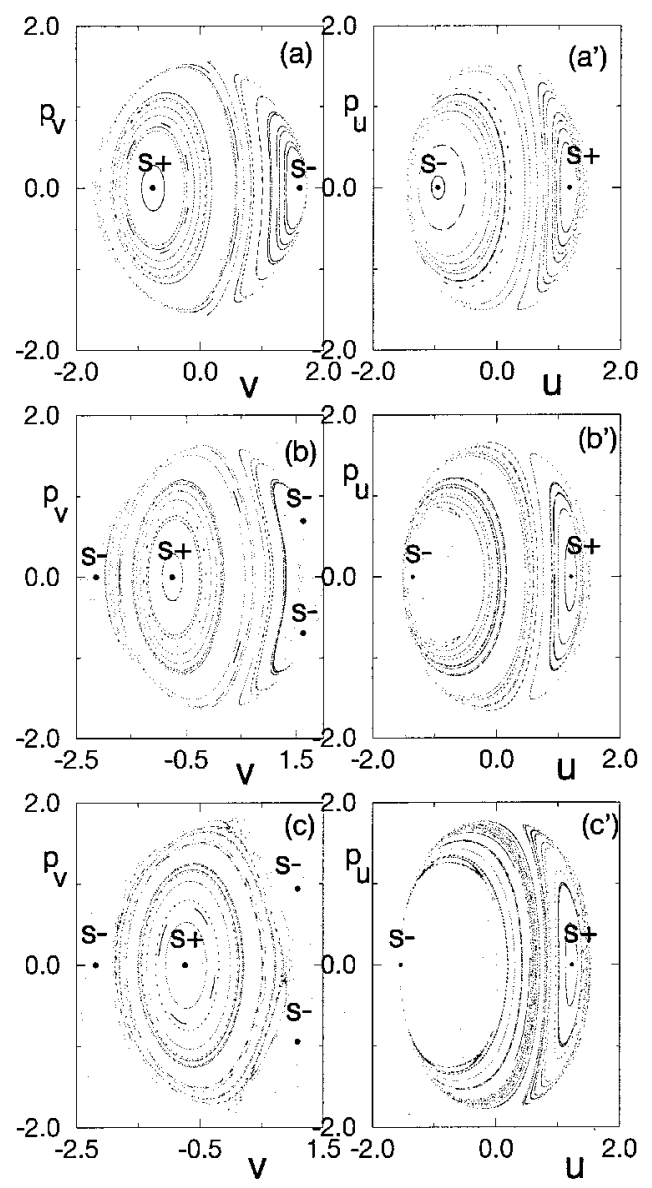

FIG. 3. A collection of surfaces of section at three different energies with fixed external fields $B=6 \mathrm{~T}, F=1000 \mathrm{~V} / \mathrm{cm}$, or $f$ $=0.2587$ : (a) $E=-250 \mathrm{~cm}^{-1}$ or $\epsilon=-1.313$ and $\omega=1.620$; (b) $E$ $=-190 \mathrm{~cm}^{-1}$ or $\epsilon=-0.998$ and $\omega=1.413$; (c) $E=-140 \mathrm{~cm}^{-1}$ or $\epsilon=-0.736$ and $\omega=1.213$.

\section{B. Two elementary periodic orbits and their role in the organization of planar motions}

For the reduced 2D Hamiltonian, there are two fundamental periodic orbits $S_{+}$and $S_{-}$, and they are "organizers" of the planar motions. These orbits were studied by Flöthmann and Welge [12], who show pictures of these orbits at various energies. $S_{+}$is nearly circular in the $(x, y)$ plane, but the circle is not centered at the nucleus. The electron runs around $S_{+}$in a counterclockwise sense, so the Coulomb force and the Lorentz force both point inwards. In the energy range we study, $S_{+}$is stable, so quasiperiodic orbits oscillate about it [13].

Figure 3 shows surfaces of section calculated in two different ways at various energies. The surfaces of section on the left are defined by $u=0$ with $\dot{u}>0$ and the surfaces of section on the right are defined by $v=0$ with $\dot{v}>0$. The smooth curves on the surface of section (SOS) correspond to regular tori and they are organized into two groups: one is centered at $S_{+}$and another is centered at $S_{-}$. However, there is no fundamental distinction between these two groups, since they are not separated by an unstable periodic orbit with a separatrix.
The origin in coordinate space $(u=0, v=0)$ directly corresponds to the $p_{u}$ or $p_{v}$ axis on the SOS, so orbits that are closed at the nucleus are orbits that begin and end on the momentum axis $p_{u}$ or $p_{v}$ on the SOS. We may focus our attention on the behavior of the loops on the left-hand side of each SOS - the loops surrounding $S_{+}$on the $u=0$ SOS or the loops surrounding $S_{-}$on the $v=0$ SOS.

\section{POINCARÉ HALF-MAP}

The conventional full Poincaré map is commonly used to study bifurcations of the closed periodic orbits $[7,10]$. On the SOS generated by the full Poincaré map, periodic orbits show up as fixed points, so they can be easily located. However, since most of the closed orbits in the crossed field system are nonperiodic, the full Poincaré map is not so helpful. In this section, we define a Poincaré half-map, which is more useful.

\section{A. Full Poincaré map}

Choosing the surface $q_{i}=0$ (i.e., either $u=0$ or else $v$ $=0)$, we define a map of the corresponding Poincare plane $\left(q_{j_{0}}, p_{j_{0}}\right) \rightarrow\left(q_{j_{1}}, p_{j_{1}}\right)$ by starting the trajectories on the surface $q_{i}=0$ with $\dot{q}_{i}>0$, integrating the $\left(q_{i}(t), q_{j}(t), p_{i}(t)\right.$, $\left.p_{j}(t)\right)$ equations, and stopping whenever the orbit passes through $q_{i}=0$ with $\dot{q}_{i}>0$ again, then recording $\left(q_{j}, p_{j}\right)$. This is called the Poincare full map $M_{p}$. We denote the pair of variables $\left(q_{j}, p_{j}\right)$ as

$$
z \equiv\left(q_{j}, p_{j}\right)
$$

and the mapping is abbreviated as

$$
z_{k+1}=M_{p}\left(z_{k} ; \epsilon, f\right) \text {. }
$$

The iterations of the Poincare full map give us the conventional Poincare SOS. Each fixed point on the SOS corresponds to a periodic orbit of the differential equations.

The surface selected above has a drawback; the trajectories do not always pass through the surface but may be tangent to it. This results in discontinuities of the map [14]. In this system, we did not find a simple Poincaré map that is entirely free of discontinuities. However, we find that the map defined in Eq. (9) is continuous in substantial regions, so we can use it to discover orderly bifurcation patterns of closed orbits.

\section{B. Poincaré half-map}

The symmetries of the system allow us to define a kind of "'half-map." If we begin trajectories at $q_{i}=0$ with $\dot{q}_{i}>0$ and stop immediately when they return to $q_{i}=0$ with $\dot{q}_{i}<0$, we call this map $M_{+}(z ; \epsilon, f)$. Similarly, if we start with $\dot{q}_{i}<0$ and stop at $q_{i}=0$ with $\dot{q}_{i}>0$, we call this map $M_{-}(z ; \epsilon, f)$. Clearly, the full Poincare map is then

$$
M_{p}(z ; \epsilon, f)=M_{-}\left(M_{+}(z ; \epsilon, f)\right) .
$$

It is not hard to show that because of the symmetries of this system, the maps $M_{+}$and $M_{-}$are related by 


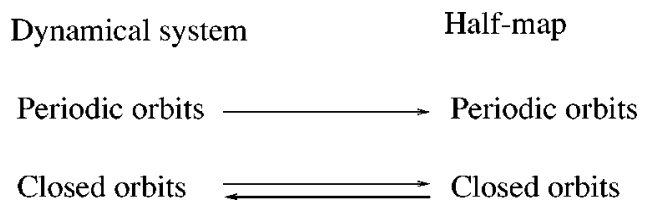

FIG. 4. Every periodic orbit of the dynamical system (defined by the differential equations) that goes through the surface of section ( $u=0$ or alternatively $v=0)$ is a periodic orbit of some iterate of the half-map. The converse is not necessarily true. Every orbit of the dynamical system that is closed at the origin $(u=0, v=0)$ is also a closed orbit of some iterate of the half-map. The converse is also true.

$$
M_{-}(z ; \epsilon, f)=-M_{+}(-z ; \epsilon, f) .
$$

Therefore,

$$
M_{p}(z ; \epsilon, f)=M_{-} M_{+}(z ; \epsilon, f)=-M_{+}\left(-M_{+}(z ; \epsilon, f)\right),
$$

that is to say, $M_{-} M_{+}=\left(-M_{+}\right)^{2}$. We define $-M_{+}(z ; \epsilon, f)$ as the Poincaré half-map.

\section{Periodic orbits of the Poincaré half-map and the winding ratio of the periodic orbits}

\section{The correspondence between periodic or closed orbits of the Poincaré half-map and periodic or closed orbits of the dynamical system}

The Poincaré half-map is defined as $-M_{+}(z ; \epsilon, f)$. Starting from any point, we may iterate the map $-M_{+}$as many times as we wish,

$$
z_{k+1}^{\prime}=-M_{+}\left(z_{k}^{\prime} ; \epsilon, f\right) .
$$

We define a periodic orbit of the half map as the set of points $\left(z_{0}, z_{1}^{\prime}, z_{2}^{\prime}, \ldots, z_{m}^{\prime}\right)$ generated according to Eq. (12) such that the $m$ th iteration returns to the initial point $z_{m}^{\prime}=z_{0}$. We define a closed orbit of the half-map as an orbit such that $q_{m}^{\prime}$ $=q_{0}=0$. (Here, as usual, "closed" means closed at the nucleus.)

Every periodic orbit of the dynamical system that goes through the surface of section is also a periodic orbit of some iterate of the half-map. Every closed orbit of the dynamical system is a closed orbit of the half-map, and vice versa (see Fig. 4).

\section{The winding ratios of the periodic orbits of the Poincaré half-map}

We focus on the loops around $S_{+}$on the $u=0$ surface to study the closed orbits. In the regular region, $S_{+}$is surrounded by smooth curves, and each curve corresponds to a regular torus in phase space.

To show how the trajectories wind around the central periodic orbit, we apply the half-map to a horizontal line to get Fig. 5. We have the following observations:

(i) Points on the SOS wind around the periodic orbit $S_{+}$, and we may say that they wind in a clockwise sense.

(ii) The winding rate decreases as we go farther from the central periodic orbit $S_{+}$. (If we travel outward from $S_{+}$on

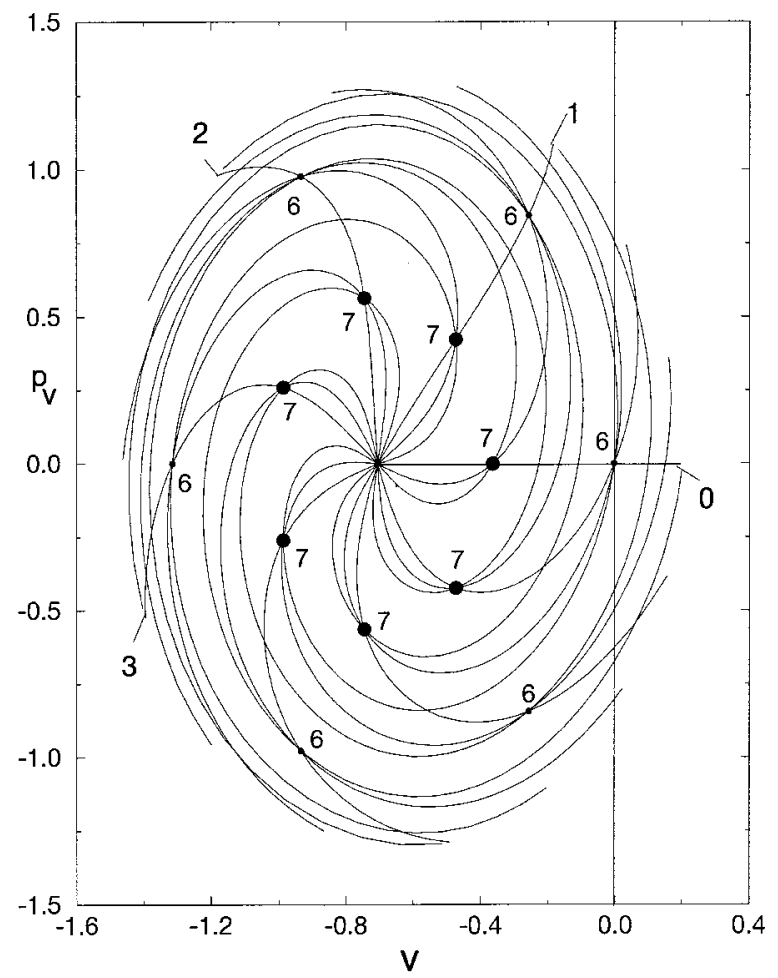

FIG. 5. The first 20 iterations of the horizontal line at $E$ $=-95 \mathrm{~cm}^{-1}$ with external fields $B=6 \mathrm{~T}$ and $F=1000 \mathrm{~V} / \mathrm{cm}$. The $5 / 7$ and $2 / 3$ periodic orbits are marked by dots and labeled by 7 and 6. We use bold $0,1,2,3$ to label the initial horizontal line and the first, second, and third iteration of the initial line, respectively.

a curve, our path bends to the left.)

(iii) Since the orbits are regular, we can relate the winding rate $\bar{\alpha}\left(\epsilon, f ; v_{0}, p_{v_{0}}\right)$ to the fundamental periods of the tori, which we choose to call $T_{u, v}\left(\epsilon, f ; v_{0}, p_{v_{0}}\right)[15]$. The winding ratio of the points is related to fundamental periods by

$$
\frac{T_{u}\left(\epsilon, f ; v_{0}, p_{v_{0}}\right)}{T_{v}\left(\epsilon, f ; v_{0}, p_{v_{0}}\right)}=\frac{\bar{\alpha}\left(\epsilon, f ; v_{0}, p_{v_{0}}\right)-\pi}{\pi}
$$

In the numerator, we subtract $\pi$ because the half-map incorporates a reflection $\left(v \rightarrow-v, p_{v} \rightarrow-p_{v}\right)$, which we regard as an advance of $\pi$ upon each return. In the denominator, we divide by $\pi$ instead of $2 \pi$ because each return to $u=0$ corresponds to a half-cycle of the $u$ motion.

(iv) Besides the central periodic orbit, $S_{+}$, there are other periodic orbits corresponding to tori with a rational period ratio. These occur when $\left[\bar{\alpha}\left(\epsilon, f, v_{0}, p_{v_{0}}\right)-\pi\right] / \pi$ is a rational fraction $m / l$. We name the torus and its periodic orbits by this rational fraction $m / l$. The periodic orbits of the map show up as intersection points of the spirals on the SOS (see Fig. 5).

(v) As energy decreases, the winding rate $\bar{\alpha}\left(\epsilon, f ; v_{0}, p_{v_{0}}\right)$ around the center $S_{+}$increases, and each rational torus moves outward from $S_{+}$. 


\section{A MODEL OF THE HALF-MAP}

In this section, we present a model Hamiltonian that generates a "twist map.' This twist map is a simple model of the half-map, and we use this twist map to illustrate the sequence of bifurcations of closed orbits. We will show in the next section that the bifurcations of closed orbits in the crossed-field system fall into the same sequence as the bifurcations in the model. Hence the model Hamiltonian provides an interpretation of our calculations on the crossed-field system.

\section{A. A model Hamiltonian}

We consider a model in which a particle moves periodically on a single coordinate $q(t)$, with an associated canonical momentum $p(t)$, so phase space is the two-dimensional plane $(q, p)$. We want a model having the following four properties: (i) the flow in phase space is steady and areapreserving, so it is governed by a time-independent Hamiltonian; (ii) the center $(q=0, p=0)$ is a fixed point, and there are no other fixed points; (iii) points nearby move in a closed curve around the center with average angular velocity $\omega_{0}$; (iv) more distant points move around with a lower average angular velocity. At a later point we will add a fifth property: one of the closed curves defines an outer boundary to the flow.

A Hamiltonian generating such a flow is

$$
H=\left(-\omega_{0} / a\right) e^{-a I},
$$

where $I=\left(p^{2}+q^{2}\right) / 4 \pi$ is the classical action. We may use $I$ and $\theta=\arctan (q / p)$ as canonical variables, and then

$$
\begin{gathered}
\dot{I}=0, \quad I=\text { const } \\
\dot{\theta}=\omega_{0} e^{-a I}, \quad \theta(t)=\theta_{0}+\omega_{0} t e^{-a I} .
\end{gathered}
$$

This is a continuous version of a "twist map" - the flow has the properties listed above. Obviously all the orbits are periodic, and any orbit that goes through some point $q_{0}$ returns repeatedly to that point (see Fig. 6).

In this model, we use the words "closed orbit" to refer to a "closed orbit at $q_{0}$ with closure time $T$ "': this is an orbit that starts at some location $q_{0}$ that is not at the center, and later, at $t=T$, returns to that same point. Let us now fix $T$, and regard $\omega_{0}$ as a variable parameter. We will speak of "bifurcations of closed orbits." By this we mean that for some fixed $q_{0}$, and some fixed $T$, as we vary the parameter $\omega_{0}$, there may be a creation or a splitting of a "closed orbit at $q_{0}$ with closure time $T$.' ' For example, if $T$ is fixed much less than $2 \pi / \omega_{0}$, then there is no closed orbit moving around the center having such a short period. However, for that fixed $T$, we can increase $\omega_{0}$ until it exceeds $2 \pi / T$, and then such a closed orbit will appear.

This looks like a completely trivial problem. In fact, however, such closed orbits appear in groups of four in an orderly sequence (a tangent bifurcation followed by a pitchfork bifurcation). After we display this behavior, we will show that planar closed orbits in the crossed-field system appear in the same way. Indeed, since the model is based on quite

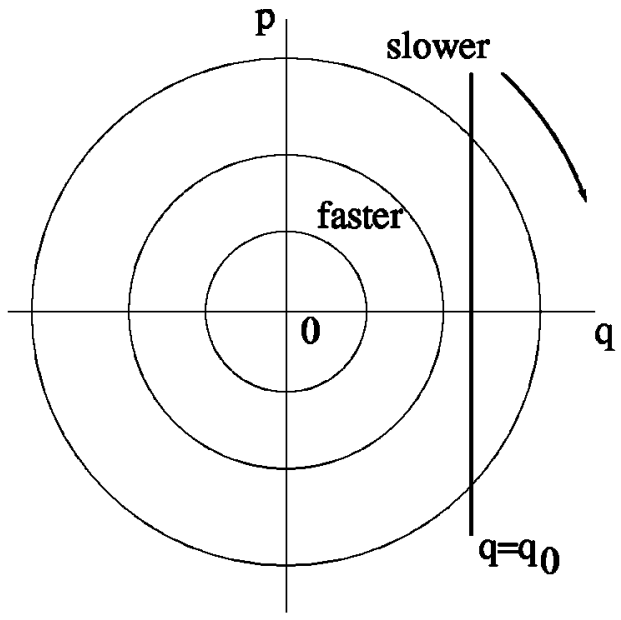

(a)
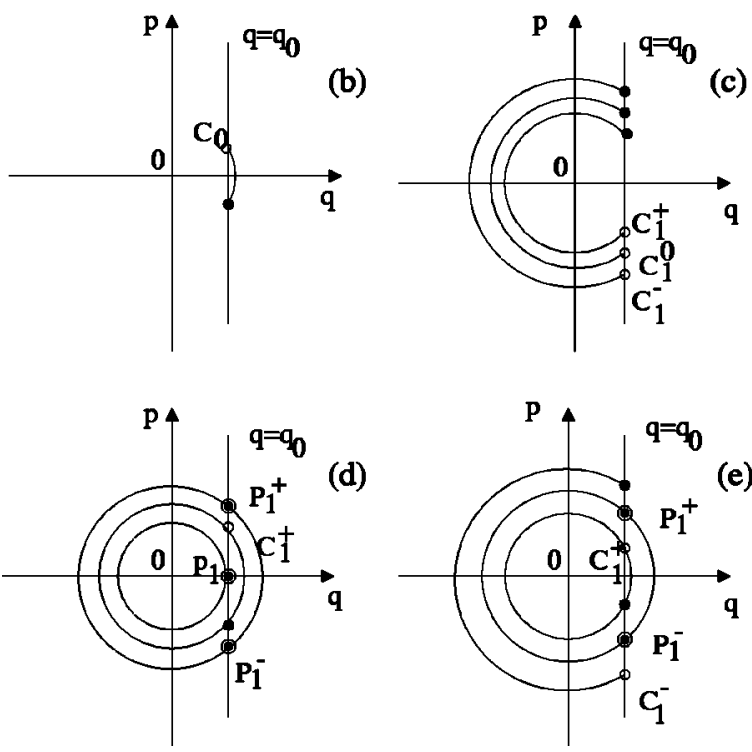

FIG. 6. (a) The motions described by the model Hamiltonian are circular rotations in the $(q, p)$ phase space. (b)-(e) The four shortest closed orbits are represented in $(q, p)$ space. Orbits begin at an open circle and end at a filled circle. (b) At small $T$ (or small $\omega_{0}$ ), only $C_{0}$ is present. (c) At some larger $T, C_{1}^{0}$ is created, and it splits into $C_{1}^{+}$and $C_{1}^{-}$. (d) $C_{1}^{+}$evolves until it becomes periodic, $P_{1}$. Then it evolves into an orbit that goes around the center more than one cycle. At the same time it splits off two periodic orbits $P_{1}^{ \pm}$that differ only in phase. (e) At the end, four closed orbits exist; two are nonperiodic, $C_{1}^{ \pm}$, and two are periodic, $P_{1}^{ \pm}$.

general assumptions, we expect that this behavior will be commonly found in physical systems.

\section{B. Normal sequence of bifurcations of closed orbits of the model Hamiltonian}

We consider the set of initial conditions ( $q=q_{0}, p=$ anything). This corresponds to a vertical line in the $(q, p)$ plane, which we call $\Gamma(0)$. Suppose we allow the points on that line to evolve under Hamiltonian's equations (15) and (16) until $t=T$. The line evolves into a curve that we call $\Gamma\left(\omega_{0} T\right)$. If the evolved curve $\Gamma\left(\omega_{0} T\right)$ intersects the 


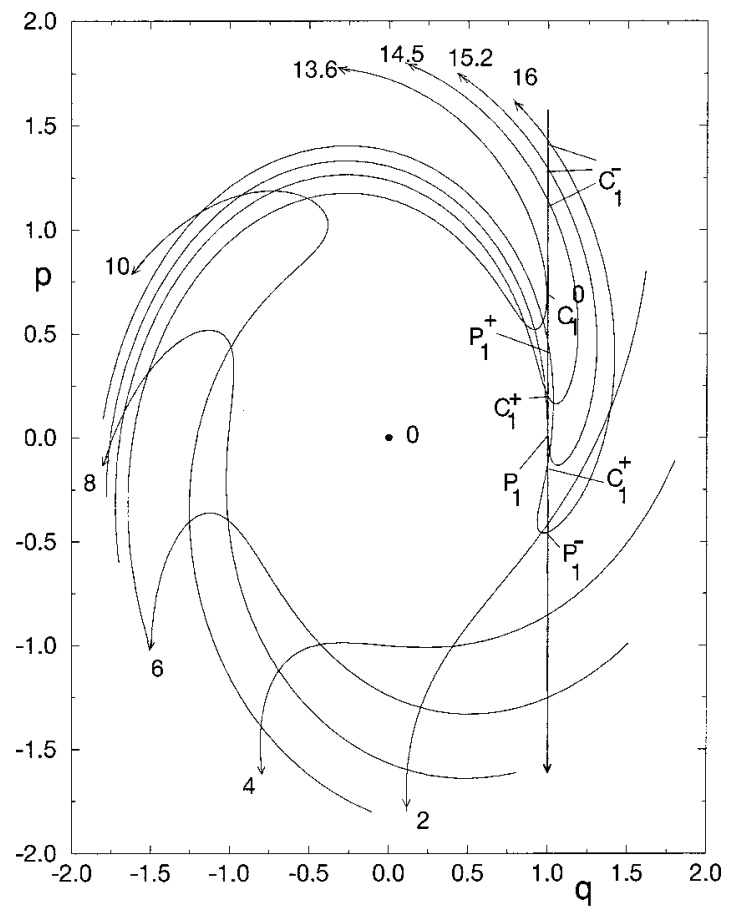

FIG. 7. Evolution of the vertical line at different times $t$ with $\omega_{0}=0.5, a=-0.2$. We label the curves by the time.

original line $\Gamma(0)$, then the intersection point is the final point of a "closed orbit with closure time $T$.",

If we fix $T$ and vary $\omega_{0}$, then the evolved curve changes in some way; it may develop new or additional intersections with the original line. These are the above-mentioned "bifurcations of closed orbits of closure time $T$.',

We need to know how the curve changes when $\omega_{0}$ changes at fixed $T$. To understand this, we only have to look at Eq. (16) and note that $\omega_{0}$ and $t$ appear together as a product. Thus to learn how the curve changes with $\omega_{0}$ at fixed $t$, we only have to ask, how does it evolve with $t$ at fixed $\omega_{0}$ ? This is shown in Fig. 7 for $\omega_{0}=0.5, a=-0.2, q_{0}=1$.

Every point moves clockwise on a circle, with points farther from the origin moving more slowly. Points at $p= \pm \infty$ do not move at all. Therefore, for small $t$, points on the lower half of the line sweep to the left, and points on the upper half of the line sweep to the right. It follows that there is just one intersection of the final curve with the initial line, and it lies in the lower-half-plane (LHP). At small $T$, there is always one orbit that moves slightly to the right of $q_{0}=1$ and then returns. We call it $C_{0}$. The zero in the subscript means that this orbit does not pass through the center $q=0$.

As $t$ increases (see Fig. 7), the curve $\Gamma\left(\omega_{0} t\right)$ evolves until eventually the "nose" of the curve is tangent to the vertical line. A new closed orbit has appeared that we name $C_{1}^{0}$; this orbit moves to the left and then returns, passing back and forth through the center $q=0$ once.

As $t$ continues to increase, the "nose" passes through the vertical line. The one closed orbit has split into two. One moves up and one moves down the vertical line, and we call the resulting orbits $C_{1}^{-}$and $C_{1}^{+}$. This sequence of events is a kind of a tangent bifurcation.

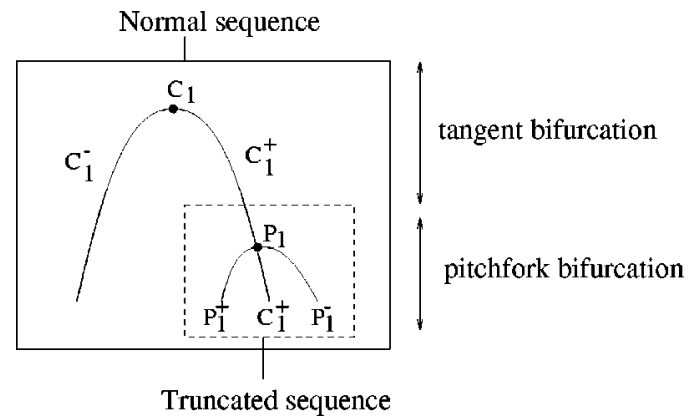

FIG. 8. The normal sequence of bifurcations: a tangent bifurcation is followed by a pitchfork bifurcation. In the truncated sequence, the tangent bifurcation is not present.

As $t$ continues to increase, the final point of $C_{1}^{+}$moves downward. To understand what happens next, consider the point $\left(q=q_{0}, p=0\right)$. This is the point on the initial line that is closest to the origin, so this point moves around the origin with the highest angular frequency,

$$
\omega_{1}=\omega_{0} \exp \left(-a q_{0}^{2} / 4 \pi\right) \text {. }
$$

When $t=2 \pi / \omega_{1}$, that point has returned to itself, and we have a periodic orbit. We see that as $t$ increases to this value, the final point of $C_{1}^{+}$moves down so that it coincides with this periodic orbit, and the formerly closed orbit $C_{1}^{+}$evolves into a periodic orbit. We may now call it $P_{1}$. The subscript means that this periodic orbit has gone around the center $(q=0)$ once.

At this point, the evolved curve $\Gamma\left(2 \pi \omega_{0} / \omega_{1}\right)$ must be tangent to the original line $q_{0}=1$ at $p=0$. As $t$ continues to increase, the part in the LHP moves to the left and the part in the upper-half-plane (UHP) moves to the right. It follows that the point of intersection with $\Gamma(0)$ moves down, and two new intersection points appear, one in the UHP and one in the LHP. The original intersection point is the continuation of $C_{1}^{+}$; it is still closed but not periodic, and we again call it $C_{1}^{+}$. The two new intersection points are periodic orbits; they both lie on the same circle and the same straight line, i.e., they are reflections of each other through the $p$ $=0$ axis. The creation of these two orbits may be called a " "pitchfork' bifurcation. In this one-dimensional model, $P^{+}$ and $P^{-}$differ only in their phase: $P^{+}$leaves $q=q_{0}$ with $\dot{q}$ $>0$, while $P^{-}$leaves with $\dot{q}<0$.

Reiterating the sequence of events, a closed orbit $C_{1}^{0}$ appears that splits into two, $C_{1}^{-}$and $C_{1}^{+} ; C_{1}^{-}$persists as a closed orbit. $C_{1}^{+}$becomes periodic (and we call it $P_{1}$ ), then splits off two periodic orbits $P_{1}^{+}$and $P_{1}^{-}$, after which it is again closed but not periodic $\left(C_{1}^{+}\right)$. Four closed orbits survive, two of which are periodic $\left(P_{1}^{+}, P_{1}^{-}\right)$and two of which are not $\left(C_{1}^{-}, C_{1}^{+}\right)$. A picture is given in Fig. 8 .

\section{The bifurcation sequences of longer trajectories}

If we iterate the vertical line for a longer time, the curve $\Gamma\left(\omega_{0} t\right)$ curls around the center a second time. Another sequence of bifurcations occurs and creates closed orbits: $C_{2}^{0}$ 


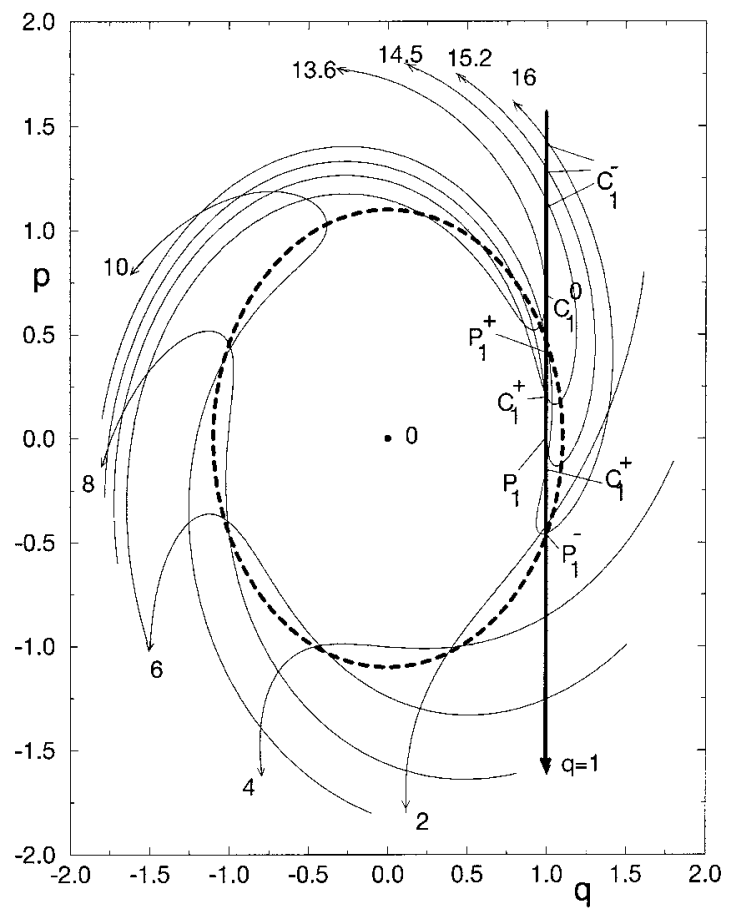

FIG. 9. Same as Fig. 7, with an outer boundary added. The dashed line is the forced boundary and the particle is only allowed to move in the inner region, so $C_{1}^{0}$ and $C_{1}^{-}$do not exist in the bounded phase space.

$\rightarrow\left(C_{2}^{-}, C_{2}^{+}\right), C_{2}^{+} \rightarrow P_{2} \rightarrow\left(P_{2}^{+}, C_{2}^{+}, P_{2}^{-}\right)$. These orbits pass back and forth the center twice $\left(P_{2}\right.$ executes two full circles around the center).

\section{Truncated sequence of bifurcations of closed orbits of the model Hamiltonian}

To understand the observed "truncated sequence" of bifurcations of closed orbits, we add one more element to our model: an outer boundary. Suppose the allowed region of phase space is bounded by a circle (see the dashed circle in Fig. 9), and the radius of this circle is too small to include the orbit $C_{1}^{0}$. Then the bifurcation sequence starts from an existing closed orbit $C_{1}^{+}$.

As energy increases, $C_{1}^{+}$first evolves into a periodic orbit, then splits into two periodic orbits and a closed orbit by the pitchfork bifurcation already discussed. The result is that three closed orbits are created in an orderly sequence of events: $C_{1}^{+}, P_{1}^{+}$, and $P_{1}^{-}$. The closed orbit $C_{1}^{-}$is missing. We may call this event of a pitchfork bifurcation without a nearby tangent bifurcation the "truncated sequence of bifurcations of closed orbits in the model system."

\section{E. Bifurcation sequence of closed orbits at fixed $t$ and varying $\omega_{0}$}

We have discussed above the bifurcation sequences of the closed orbits at fixed $\omega_{0}$ and varying $t$. Since $t$ and $\omega_{0}$ are interchangeable in Eq. (16), fixing $t$ and varying $\omega_{0}$ is equivalent to fixing $\omega_{0}$ and varying $t$. Therefore, the bifurcation sequences we discussed above also happen as we fix $t$ and vary $\omega_{0}$. The pitchfork bifurcation for the closed orbits that pass the origin back and forth $n$ times happens when $\omega_{0}>2 \pi n / T$ and $\omega_{n}=\omega_{0} e^{-a q_{0}^{2} / 4 \pi}=2 \pi n / T$, where $\omega_{n}$ is the winding rate of the point $\left(q=q_{0}, p=0\right)$.

\section{F. Local bifurcations versus regional sequences of bifurcations}

As discussed earlier, mathematical bifurcation theory is focused on "local" properties of bifurcations [10]. The theorems describe what happens within an arbitrarily small neighborhood of a point in phase space in an arbitrarily small region of parameter space. From this perspective, the abovedescribed tangent bifurcation and pitchfork bifurcation are independent events that have no relationship to each other. Also, from the mathematical perspective, the pitchfork bifurcation is considered exceptional ("nongeneric").

A physicist's perspective is different. For logical reasons we observe a tangent bifurcation and a pitchfork bifurcation close to each other, but not within an arbitrarily small neighborhood. Accordingly, to distinguish this behavior from the local properties of each bifurcation, we will call the pair of bifurcations a "regional" sequence. Implicit in this discussion is the view that in systems of interest in physics, this connected pair of bifurcations will commonly be found.

On a larger scale in parameter space, one regional bifurcation sequence may be connected with another; we call these "large-scale connections." If one were ever to work out the whole family of closed orbits and their bifurcations in all of phase space and/or all of parameter space, we would have a "global" picture.

In the crossed-field system, we will examine several regional sequences of bifurcations, and a few large-scale connections.

\section{LOCAL BIFURCATIONS AND REGIONAL SEQUENCES OF BIFURCATIONS IN THE CROSSED-FIELD SYSTEM}

Now we return to the crossed-field system, and we show that what we learned about the model also applies to our real system. From the above, we see that both the "normal" and "truncated" bifurcation sequences have periodic orbits involved. This means that the bifurcation sequence includes the passage of a rational torus through the line of initial conditions. Therefore, in this section, we first examine the evolution of rational tori; then we discuss the bifurcations of the closed orbits. In this section, we focus on local bifurcations and regional sequences of bifurcations.

\section{A. Creation and evolution of rational tori}

In this system, we cannot get the whole picture by looking at just one Poincaré section. We must examine $u=0$ and $v$ $=0$ sections in overlapping ranges of energy. (The problem is that the pitchfork bifurcations often occur when the closed orbit has a point of tangency with a SOS.)

In Fig. 10, we show the evolution of the rational torus $2 / 3$ on both $\left(u, p_{u}\right)$ and $\left(v, p_{v}\right)$ surfaces. On the $\left(v, p_{v}\right)$ plane, the winding ratio decreases with increasing distance from the center $S_{+}$, and as energy decreases, the winding ratio around 


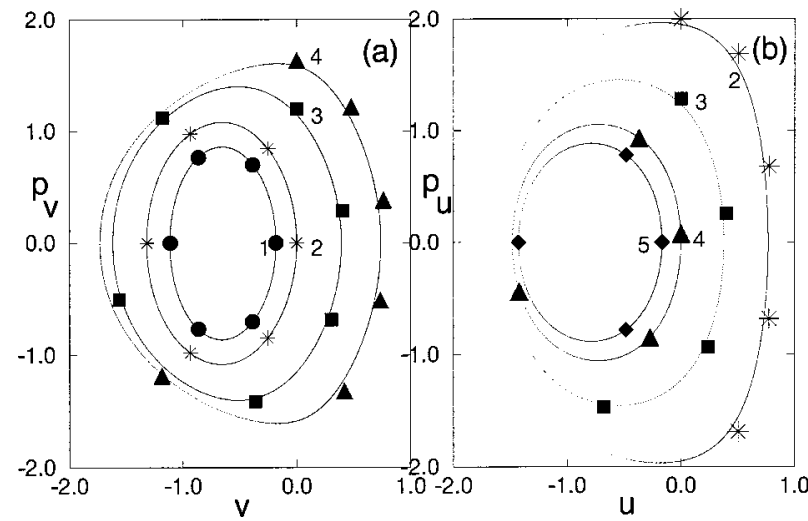

FIG. 10. The $2 / 3$ rational torus in $\left(v, p_{v}\right)$ and $\left(u, p_{u}\right)$ planes in overlapping ranges of energy. In (a), from inner to outer (curve 1 to curve 4), energy decreases and the energies are $-70 \mathrm{~cm}^{-1},-95.3$ $\mathrm{cm}^{-1},-160 \mathrm{~cm}^{-1}$, and $-212 \mathrm{~cm}^{-1}$. In (b), from outer to inner (curve 2 to curve 5), energy also decreases; the energies are -95.3 $\mathrm{cm}^{-1},-160 \mathrm{~cm}^{-1},-212 \mathrm{~cm}^{-1}$, and $-230 \mathrm{~cm}^{-1}$.

the center $S_{+}$increases. It follows that as energy decreases, the rational torus $2 / 3$ moves outward. At some critical energy, it becomes tangent to the $p_{v}$ axis. [See curve 2 in Fig. 10(a).]

This indicates that one of the members on this rational torus passes through the origin and becomes a periodic orbit closed at the origin. We call the energy at which the rational torus $m / n$ is tangent to the $p_{v}$ axis $E_{m, n}^{+}$. When energy continues to decrease, the rational torus $2 / 3$ continues to expand so that it crosses the $p_{v}$ axis, and two different periodic closed orbits are created. As energy further decreases, at some critical energy it touches the classical boundary in the $\left(v, p_{v}\right)$ plane, and then it disappears from this plane. We call the critical energy at which the rational torus $m / n$ disappears from this plane $E_{m, n}^{-}$. To see what is happening there, it is better to look at the other SOS $\left(u, p_{u}\right)$.

On the $\left(u, p_{u}\right)$ plane, the winding ratio increases with increasing distance from the center $S_{-}$, and as energy increases, the winding ratio around the center $S_{-}$decreases. When energy is higher than $E_{2,3}^{+}$, the rational torus $2 / 3$ does not appear on the $\left(u, p_{u}\right)$ surface. At $E=E_{2,3}^{+}$, it appears at the boundary. After that, as energy decreases, it moves toward the center $S_{-}$. The two crossings of the rational torus on the $p_{u}$ axis indicate again that two members of the periodic family $2 / 3$ are closed at the origin. At $E=E_{2,3}^{-}$, the rational torus $2 / 3$ becomes tangent to the $p_{u}$ axis, and two periodic closed members merge to one periodic closed member. As energy goes below $E=E_{2,3}^{-}$, the rational torus moves away from the $p_{u}$ axis and toward the center $S_{-}$. [See curve 5 in Fig. 10(b).] Finally it disappears at the center $S_{-}$.

Based on the evolution of the rational torus $2 / 3$, we can reiterate the evolution of a general rational torus $m / n$. At some high energy, the rational torus $m / n$ is created at $S_{+}$, and as energy decreases, it moves away from $S_{+}$. At $E$ $=E_{m, n}^{+}$, it becomes tangent to the $p_{v}$ axis and one periodic closed orbit is created; at that point it also becomes visible in the other SOS. As energy continues to decrease, this rational torus crosses the momentum axes on either SOS, and two members of the periodic family $m / n$ are closed at the origin. At $E=E_{m, n}^{-}$, the rational torus becomes tangent to the $p_{u}$ axis and two periodic closed members merge to one periodic closed member. As energy further decreases, the rational torus moves away from the $p_{u}$ axis and toward $S_{-}$; it has then disappeared from the $\left(v, p_{v}\right)$ plane. Finally, this rational torus is annihilated at $S_{-}$. We use the names of the rational tori $(\mathrm{m} / \mathrm{n})$ to name the associated closed orbits such as $C_{2,3}^{+}$; the family name $(2,3)$ is related to the winding ratio of the periodic members of the family.

\section{B. The sequence of the bifurcation patterns of closed orbits}

The evolution of the rational torus shows that the bifurcations of the periodic closed orbits $m / n$ happen at $E_{m, n}^{+}$and $E_{m, n}^{-}$. In this section, we show that both of the bifurcation sequences illustrated by the model Hamiltonian happen in the crossed-field system: we have observed some normal sequences by iterating the $p_{v}$ axis in the $\left(v, p_{v}\right)$ plane near $E_{m, n}^{+}$and we have observed some truncated sequences by iterating the $p_{u}$ axis in the $\left(u, p_{u}\right)$ plane near $E_{m, n}^{-}$.

\section{1. "Normal"' bifurcation sequences}

The $(2,3)$ bifurcation is observed in the sixth iterate of the half-map in the $\left(p_{v}, v\right)$ plane. The starting points lie on the $p_{v}$ axis. Figure 5 shows that $E_{2,3}^{+} \approx-95 \mathrm{~cm}^{-1}$. We iterate the $p_{v}$ axis on the $u=0$ surface over the energy range $-50 \mathrm{~cm}^{-1}$ to $-105 \mathrm{~cm}^{-1}$ to observe the $(2,3)$ "normal' bifurcation sequence.

The result is shown in Fig. 11. When energy is below 60.5 $\mathrm{cm}^{-1}$, the sixth iteration does not touch the momentum axis. At the energy $E=60.5 \mathrm{~cm}^{-1}$, it is tangent to the $p_{v}$ axis and one closed orbit $C_{2,3}^{0}$ is created [see Fig. 11(a)]. As we continue to decrease the energy, the sixth iteration crosses the $p_{v}$ axis, and the closed orbit $C_{2,3}^{0}$ splits into two closed orbits $C_{2,3}^{+}$and $C_{2,3}^{-}$[see Fig. 11(b)]. This sequence of events is the tangent bifurcation defined in Sec. V B. Then the closed orbit $C_{2,3}^{+}$moves toward the origin and becomes a periodic orbit $P_{2,3}$ at the critical energy $\left(E_{2,3}^{+}=-95.3 \mathrm{~cm}^{-1}\right)$ of the $2 / 3$ rational torus. As the energy passes through $E_{2,3}^{+}$, the periodic orbit $P_{2,3}$ returns to closed orbit $C_{2,3}^{+}$and two periodic orbits $\left(P_{2,3}^{+}, P_{2,3}^{-}\right)$are created. The creation of these two periodic orbits is the "pitchfork bifurcation" defined in Sec. $\mathrm{VB}$. These two periodic orbits are related to each other by $P_{2,3}^{-}=T \sigma_{u} P_{2,3}^{+}$.

Figure 12 shows the normal bifurcation sequence of the $(5,7)$ family of orbits, which appears on the seventh iterate of the half-map near $E_{5,7}^{+}=-135 \mathrm{~cm}^{-1}$. Since it is an oddnumber iteration, the periodic orbits involved in this bifurcation sequence are periodic orbits of the half-Poincare map and each of them is half of a periodic orbit of the dynamical system.

These bifurcation sequences are the "normal" bifurcation sequence in which the closed orbits are created in a group of four: $C_{m, n}^{-}, C_{m, n}^{+}, P_{m, n}^{+}$, and $P_{m, n}^{-}$. As stated earlier, $(m, n)$ means that periodic members have a rational winding ratio 

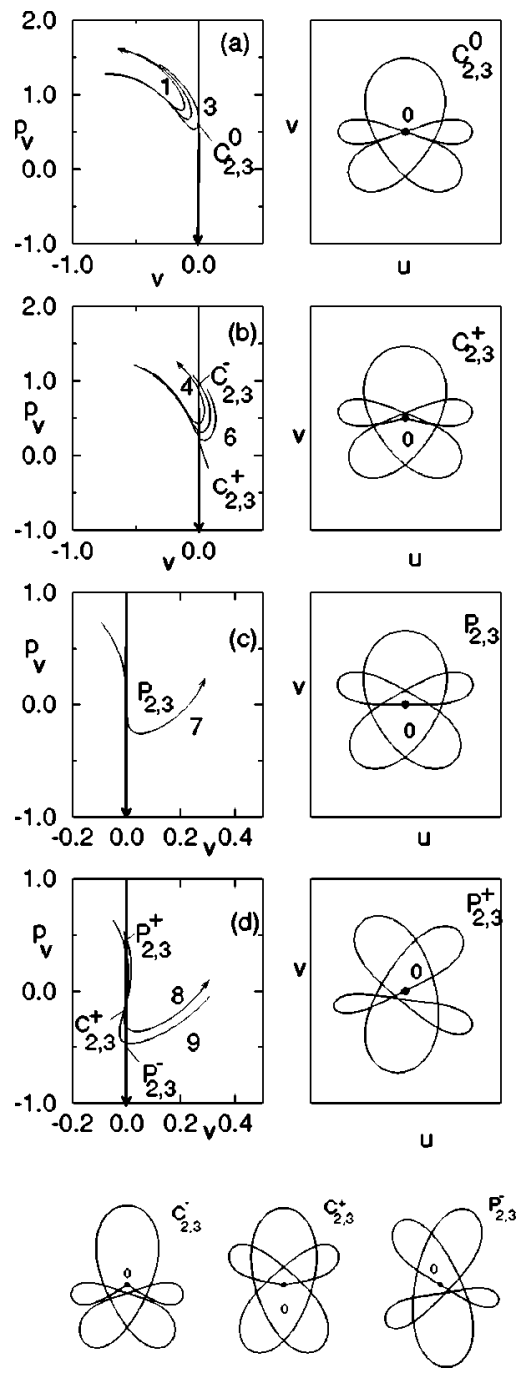

FIG. 11. The sixth iteration of the bold vertical arrow line with external fields $B=6 \mathrm{~T}$ and $E=1000 \mathrm{~V} / \mathrm{cm}$ at various energies: (a) $E=-50,-55,-60.5\left(\mathrm{~cm}^{-1}\right)$ from 1 to 3 ; (b) $E=-65,-70,-75$ $\left(\mathrm{cm}^{-1}\right)$ from 4 to 6 ; (c) $E=-95.3\left(\mathrm{~cm}^{-1}\right)$ labeled 7 ; (d) $E$ $=-100,-105\left(\mathrm{~cm}^{-1}\right)$ from 8 to 9 .

$m / n$, and the closed orbits lie on tori having winding ratios close to this rational number.

\section{2. "Truncated" bifurcation sequence}

The truncated $(2,3)$ bifurcation sequence is observed in the fourth iterate of the half-map on the $\left(u, p_{u}\right)$ plane. $E_{2,3}^{-}$ $\approx-212 \mathrm{~cm}^{-1}$; i.e., as we go to energy lower than $E_{2,3}^{-}, P_{2,3}$ is destroyed. Therefore, we start from below and increase $E$ to observe the creation of the closed orbits. Figure 13 shows the fourth iteration of the momentum axis. Curve 1 corresponds to $E=-220 \mathrm{~cm}^{-1}$. It crosses the momentum axis once, but in contrast to curve 1 in Fig. 11, it does not curve back and cross the momentum axis a second time. Compared to our model in Sec. VD, we could explain this by saying that the SOS has an outer boundary, on which there is a finite positive winding rate. If the arrowhead in Fig. 13 were extended to the outer boundary, it would still map around to the positive quadrant. Hence there is one closed orbit $C_{2,3}$ and
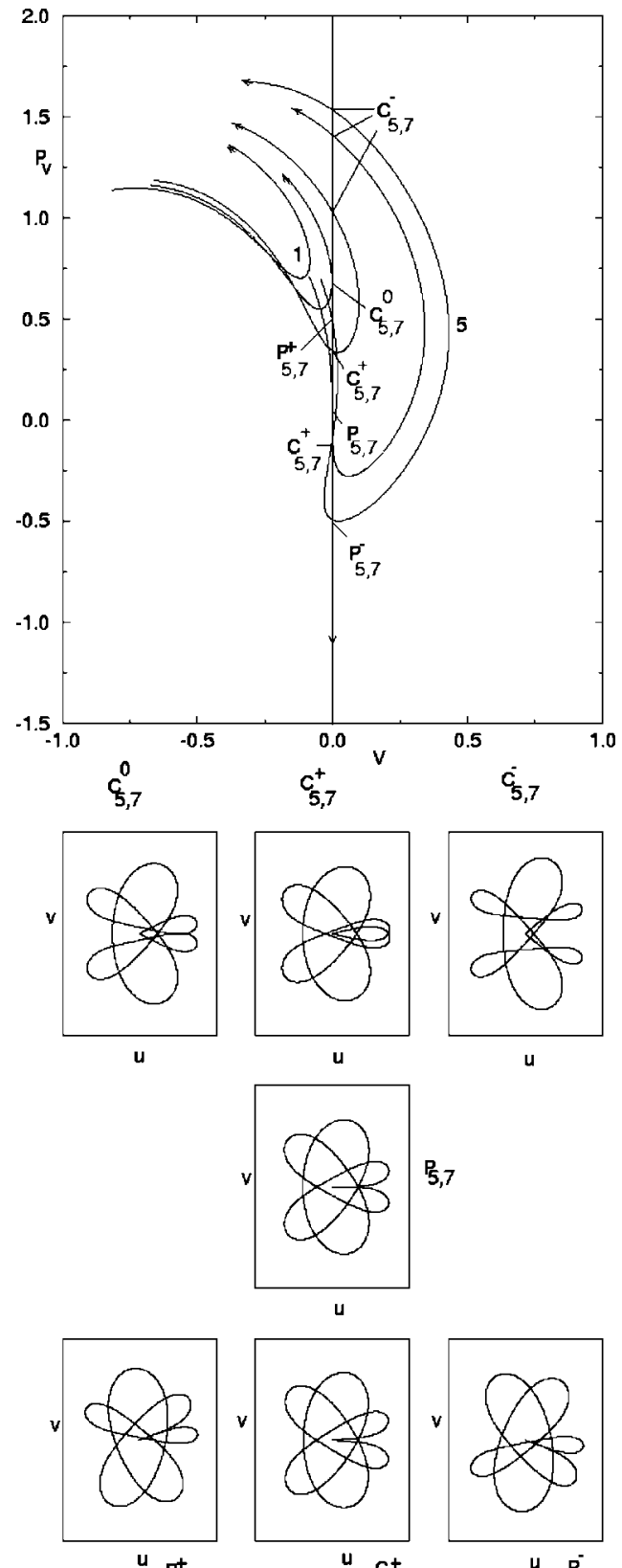

$\mathbf{P}_{5,7}^{+}$

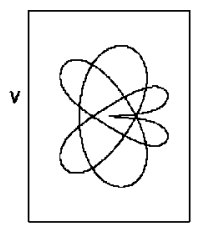

$\mathrm{C}^{+}$

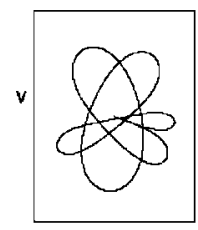

u $\quad P_{5,7}^{-}$

FIG. 12. The seventh iteration of the bold vertical arrow line with external fields $B=6 \mathrm{~T}$ and $E=1000 \mathrm{~V} / \mathrm{cm}$ at various energies: $E=-95,-101.5,-110,-135,-145\left(\mathrm{~cm}^{-1}\right)$ from 1 to 5 .

this closed orbit moves toward the origin as energy increases. At $E=E_{2,3}^{-}, C_{2,3}$ becomes a periodic orbit $P_{2,3}$. Then the periodic orbit bifurcates into one closed orbit $C_{2,3}$ and two periodic orbits $\left(P_{2,3}^{+}, P_{2,3}^{-}\right)$as energy goes above $E_{2,3}^{-}$. Thus we have only the pitchfork bifurcation in which a closed orbit creates a pair of periodic closed orbits. The two new orbits are related by $P_{2,3}^{-}=T \sigma_{v} P_{2,3}^{+}$.

The orbit $C_{2,3}$ is not the same as $C_{2,3}^{ \pm}$, discussed in the preceding section (these orbits do not continuously connect to each other). The orbits $C_{3,5}$ and $P_{3,5}^{ \pm}$shown in Fig. 2 are a second example of the truncated sequence. If the third iterate of the $p_{u}$ axis is shown at energies near $-186 \mathrm{~cm}^{-1}$, we get 

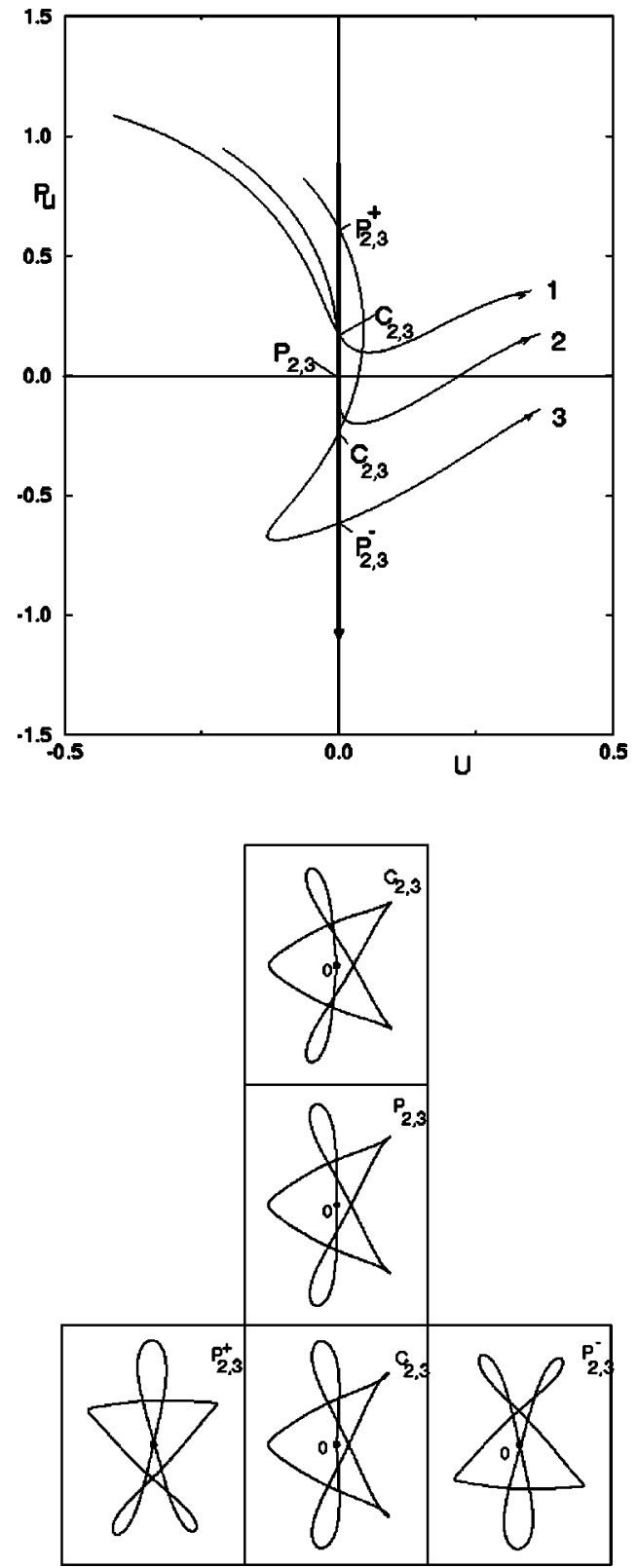

FIG. 13. The fourth iteration of the bold vertical arrow line with external fields $B=6 \mathrm{~T}$ and $E=1000 \mathrm{~V} / \mathrm{cm}$ at various energies: $E$ $=-220,-212,-200\left(\mathrm{~cm}^{-1}\right)$ from 1 to 3 . Below are shown the resulting orbits. (In the pictures of $P_{2,3}^{ \pm}$, the nucleus is close to, but not quite at, the location of a self-intersection of the orbit. Compare Fig. 11.)

a picture comparable to Fig. 13. In this case $P_{3,5}^{-}=T \sigma_{u} P_{3,5}^{+}$.

Hence we observe that both "normal" and "truncated", bifurcation sequences appear in the crossed-field system. The "normal" bifurcation sequence involves the bifurcation of a periodic closed orbit at $E_{m, n}^{+}$, and the "truncated" bifurcation sequence involves the bifurcation of a periodic closed orbit at $E_{m, n}^{-}$.

\section{SOME LARGE-SCALE CONNECTIONS OF REGIONAL BIFURCATIONS}

In the local and regional bifurcations, we observed the creation of the closed orbits $C_{m, n}^{-}, C_{m, n}^{+}, P_{m, n}^{+}, P_{m, n}^{-}$. Now we

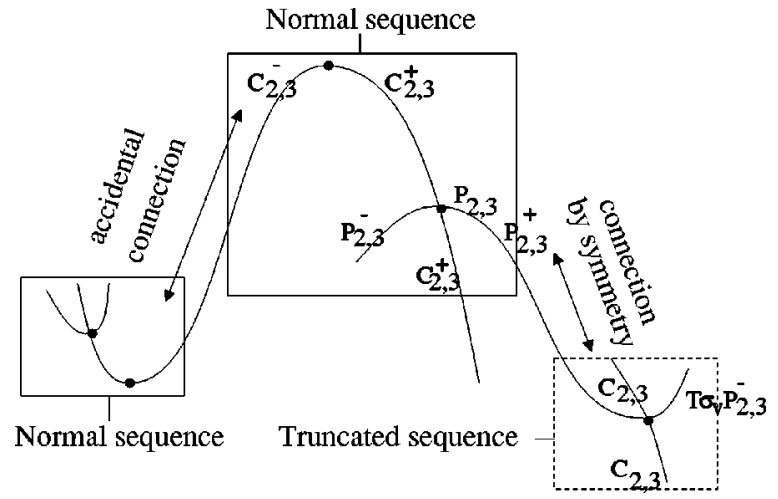

FIG. 14. We show large-scale connections of two normal regional bifurcation sequences and one truncated regional bifurcation sequence. The central normal sequence is connected to the left normal sequence by an "accidental" connection and is connected to the truncated sequence by the symmetries of the system.

ask what happens after they are created. To answer this question, we studied the evolution of the closed orbits after their creation and found that one closed orbit created in one regional bifurcation sequence may evolve and get involved in another regional bifurcation sequence. Hence the regional bifurcation sequences are connected with each other. We call a bifurcation sequence that includes more than one regional bifurcation sequence a large-scale connection of regional bifurcations. We found that some of the large scale connections are related to the symmetry of the regularized Hamiltonian.

In Fig. 14, we show large-scale connections of three regional bifurcation sequences. When we follow the orbits in the $C_{2,3}^{+}$family to lower energies, we find that $P_{2,3}^{+}$is connected to a truncated sequence of bifurcations. As stated earlier, the closed orbit $C_{2,3}$ involved in the low-energy bifurcation of $P_{2,3}$ does not connect continuously to either of the closed orbits $C_{2,3}^{ \pm}$that are involved in the high-energy bifurcation of $P_{2,3}$. In these two regional bifurcation sequences, the periodic orbits are all connected by symmetry. The symmetry operations $T \sigma_{u}, T \sigma_{V}$, and $\sigma_{u} \sigma_{v}$ acting on $P_{2,3}^{+}$generate a family of four periodic orbits, which are connected to $C_{2,3}^{ \pm}$and to $C_{2,3}$ as indicated in Fig. 15 .

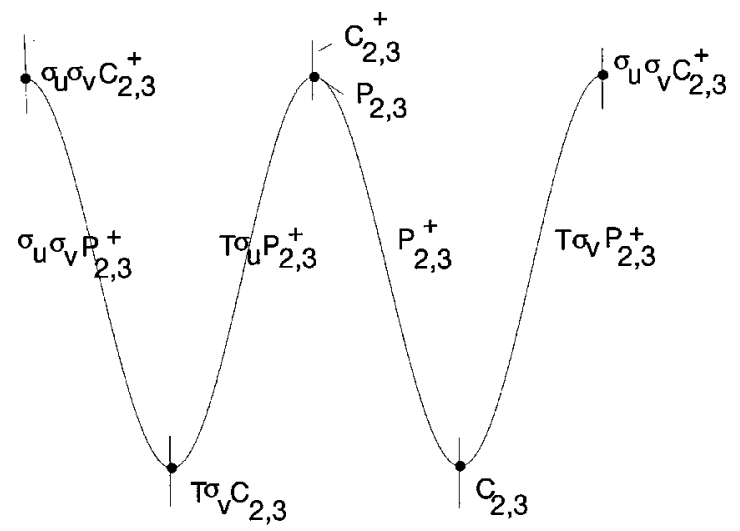

FIG. 15. We show a "unit cell" of symmetry-related large-scale connections involving $P_{2,3}^{+}$. 
We also find that $C_{2,3}^{-}$is connected to another normal sequence of bifurcations. We call this "accidental connection" since none of the trajectories in the other sequence is connected to the trajectories in the $(2,3)$ sequence by the symmetry operations.

\section{CONCLUSION}

We have shown that closed orbits in the crossed-field system are created in orderly sequences of bifurcations, either a tangent bifurcation followed by a pitchfork bifurcation or a pitchfork bifurcation by itself. The pitchfork bifurcation always involves an orbit that is both closed and periodic. Periodic orbits can be identified by their winding ratio $m / l$, so we label them as $P_{m, l}$. Each such orbit has partners generated by the symmetry operations (e.g., $\sigma_{u} \sigma_{v} P_{m, l}^{+}$). The closed orbits that are connected with $m / l$ periodic orbits are labeled $C_{m, l}$ or $C_{m, l}^{ \pm}$. At the pitchfork bifurcation, $C_{m, l}$ or $C_{m, l}^{+}$coincides with the periodic orbit $P_{m, l}$. Study of a model Hamiltonian indicates that closed orbits in any integrable system should have comparable behavior.

\section{ACKNOWLEDGMENT}

The authors thank the NSF for financial support.

\section{APPENDIX: PITCHFORK BIFURCATIONS OF CLOSED ORBITS ARE GENERIC IN INTEGRABLE HAMILTONIAN SYSTEMS}

We have shown that both in the model system defined in Sec. V and in the crossed-field system, we typically see a tangent bifurcation and a pitchfork bifurcation in an orderly sequence; often we see a pitchfork bifurcation by itself, with no nearby tangent bifurcation. This behavior may sound strange to those who are familiar with the theory of bifurcations of periodic orbits. Tangent bifurcations of periodic orbits are generic, but pitchfork bifurcations are not [9]. Nevertheless, in our calculations we have come across pitchforks more frequently than tangents.

One might expect that this is connected to symmetries of the system. It has been shown that pitchfork bifurcations of periodic orbits are generic if the Hamiltonian has a reflection symmetry [1]; if this symmetry is broken, then the pitchfork bifurcations are converted to tangent bifurcations. All of the pitchfork bifurcations of closed orbits that we have calculated in the crossed-field system are connected with a symmetry, and that leads one to suspect that breaking the symmetry would have the same effect. We therefore carried out some numerical experiments on simple maps to examine this symmetry breaking. In these calculations, pitchfork bifurcations turned out to be unexpectedly robust; they were not destroyed when the symmetry was broken.

We propose below that pitchfork bifurcations of closed orbits represent a generic pattern of behavior in integrable Hamiltonian systems. We will illustrate this by examining the time evolution of a line in the phase plane. Our mathematics will be informal and intuitive rather than formal and rigorous [16].

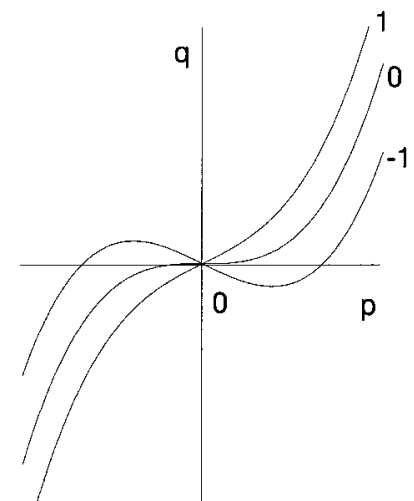

Pitchfork bifurcation

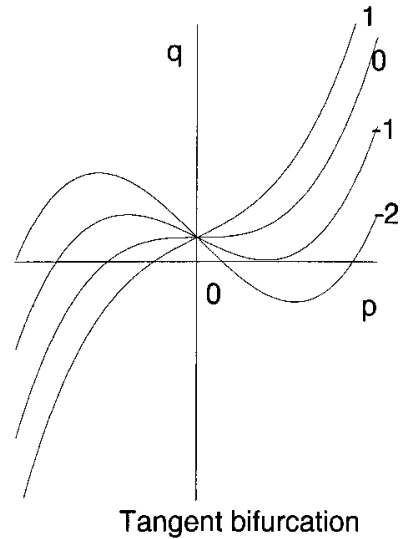

Tangent bifurcation
FIG. 16. Evolution of a cubic curve $q(p)=a+b p+p^{3}$ at fixed $a$ and various $b$. Left: $a=0$ gives a pitchfork bifurcation; right: $a$ $\neq 0$ gives a tangent bifurcation.

\section{Generic bifurcations in cubic curves}

A general cubic curve can be expressed by the function

$$
Q(P)=A+B P+C P^{2}+D P^{3} .
$$

The family of cubic curves corresponds to the family of maps $R \times R^{4} \rightarrow R$ defined by Eq. (A1). For almost all values of the parameters $A \cdots D$, the cubic has either one or three real roots. A bifurcation is a change in the number of real roots as the parameters change.

The parameter space can be reduced to two significant dimensions. For $D \neq 0$, we can divide by $D, q=Q / D$, without changing the number of real roots, and we can also translate the origin of the $\mathrm{P}$ coordinate $p=P-C / 3$ to eliminate the quadratic term. Thus by a translation in $p$ and a stretch in $q$, we can reexpress the cubic function as

$$
q(p)=a+b p+p^{3} .
$$

If $a=0$, then by varying the parameter $b$ from positive to negative, we get a pitchfork bifurcation (Fig. 16): $p=0$ is always a root, and when $b$ passes through zero, two new roots are created, one on either side of $p=0$. However, for all other values of the parameter $a$, we get a tangent bifurcation: for $a>0$ and $b>0$, only the negative root is present, and when $b$ decreases to a sufficiently negative value, two new roots are created at positive $x$. Thus in the twodimensional parameter plane $(a, b)$, tangent bifurcations are generic, and pitchfork bifurcations occur only on the line $a$ $=0$, a set of measure zero in the plane.

Returning to the four-dimensional parameter space, suppose the parameters $A \cdots D$ vary in some general manner as a function of some other single parameter, which we will call $t$. Examining Fig. 16, we see that the only way we can get a pitchfork bifurcation is if the parameters $A(t) \cdots D(t)$ vary in such a way that for some value $t=\tilde{t}$, there exists a value $p=\tilde{p}$ such that $q$ and its first two derivatives with respect to $p$ all vanish simultaneously:

$$
\begin{array}{r}
q(\tilde{p} ; \tilde{t})=0, \\
q^{\prime}(\tilde{p} ; \tilde{t})=0,
\end{array}
$$




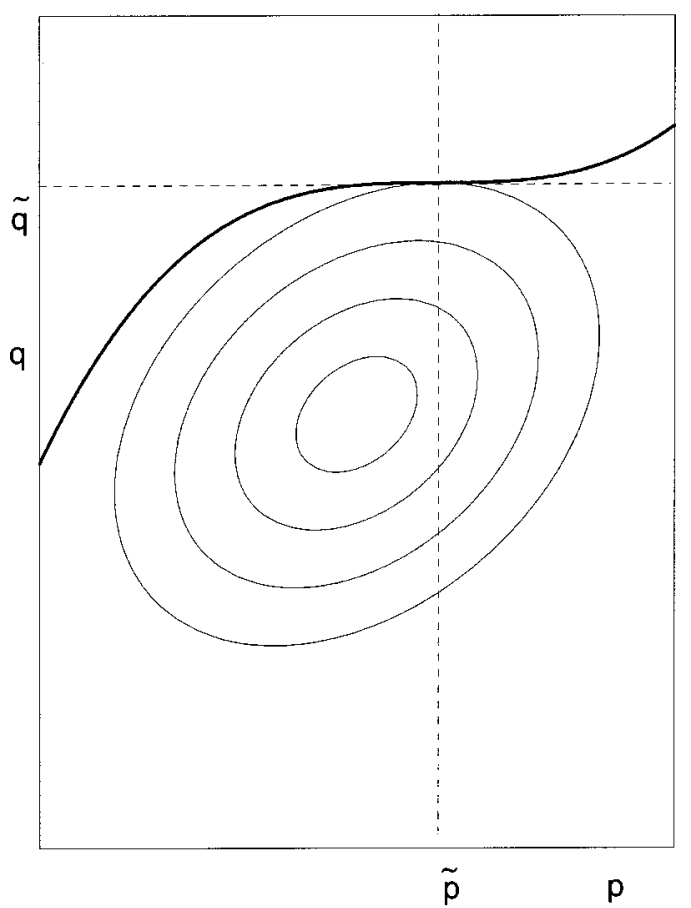

FIG. 17. Contours of a general Hamiltonian function $h(p, q)$, the line $q=\tilde{q}$, and the evolution of that line to $t=\tilde{t}$.

$$
q^{\prime \prime}(\tilde{p} ; \tilde{t})=0
$$

We will show that this seemingly exceptional condition occurs commonly in integrable Hamiltonian systems; i.e., pitchfork bifurcations are generic one-parameter bifurcations of closed orbits in integrable Hamiltonian systems.

\section{Proof in a special case}

We consider a system with one degree of freedom, having a Hamiltonian $h(p, q)$ that generates equations of motion

$$
\begin{gathered}
\dot{q}=\partial h / \partial p, \\
\dot{p}=-\partial h / \partial q .
\end{gathered}
$$

If $h(p, q)$ is smooth, then the solutions $\left(q\left(t ; p_{0}, q_{0}\right), p\left(t ; p_{0}, q_{0}\right)\right)$ are smooth functions of $t$ and of the initial conditions $\left(p_{0}, q_{0}\right)$. We assume that in a domain of the phase plane, the contours of $h(p, q)$ form smooth closed curves (Fig. 17).

We take an arbitrary line $q=\tilde{q}$ in this domain, and we examine its time evolution. Typically there is some value $\tilde{h}$ such that the contour $h(p, q)=\widetilde{h}$ is tangent to the line $q=\tilde{q}$; let us call the value of $p$ at the point of tangency $\tilde{p}$. The initial point $\left(q_{0}=\tilde{q}, p_{0}=\tilde{p}\right)$ evolves with time so that it moves around the contour $h(p, q)=\widetilde{h}$, and it eventually returns to the initial point at a time we call $t=\tilde{t}$. Similarly, every point on the initial line $\left(q_{0}=\tilde{q}, p_{0}=\right.$ anything $)$ evolves to the curve $\left[q\left(\tilde{t} ; p_{0}, \tilde{q}\right), p\left(\tilde{t} ; p_{0}, \tilde{q}\right)\right]$. We hold $\tilde{t}$ and $\tilde{q}$ fixed, so $p_{0}$ is the parameter defining the curve.

Proposition. At that value $t=\tilde{t}$,

$$
\begin{gathered}
q=\tilde{q}, \\
\partial q / \partial p_{0}=0, \\
\partial^{2} q / \partial p_{0}^{2}=0, \\
\partial p / \partial p_{0}=0 .
\end{gathered}
$$

Equation (A8) is a trivial statement: we defined $\tilde{t}$ so that it would be true. Equations (A9) and (A10) are not quite so obvious. Comparing them with Eqs. (A3)-(A5), we see that these equations are precisely what is needed to ensure that we get a pitchfork bifurcation of the closed orbit. Equation (A11) is an extra condition that is needed in order to prove Eq. (A10).

Proof. (i) Since the contour $h(q, p)$ is tangent to the line $q=\tilde{q}$ at $p=\tilde{p}$, the gradient of $h(p, q)$ is perpendicular to the line at that point,

$$
\partial h(p, q) /\left.\partial p\right|_{\tilde{p}, \tilde{q}}=0 .
$$

(ii) As time evolves, the value of $h(p, q)$ is conserved, so

$$
h\left(p\left(\tilde{t} ; p_{0}, q_{0}\right), q\left(\tilde{t} ; p_{0}, q_{0}\right)\right)=h\left(p_{0}, q_{0}\right) .
$$

Differentiating this equation once with respect to $p_{0}$, we obtain

$$
(\partial h / \partial p)\left(\partial p / \partial p_{0}\right)+(\partial h / \partial q)\left(\partial q / \partial p_{0}\right)=\left(\partial h / \partial p_{0}\right) .
$$

Evaluating this equation at $(\tilde{p}, \tilde{q})$ and using Eq. (A12), we find

$$
\left(\partial q / \partial p_{0}\right)=0
$$

(iii) Similarly, differentiating Eq. (A13) with respect to $q_{0}$, we find

$$
\left(\partial q / \partial q_{0}\right)=1 .
$$

(iv) The map is area-preserving, so

$$
\partial(p, q) / \partial\left(p_{0}, q_{0}\right)=1,
$$

and combining this with Eqs. (A15) and (A16), we find

$$
\left(\partial p / \partial p_{0}\right)=1
$$

(v) Finally, we differentiate Eq. (A13) a second time with respect to $p_{0}$, and evaluate the result at $(\tilde{p}, \tilde{q})$. Throwing away all the terms that vanish, we are left with

$$
(\partial h / \partial q)\left(\partial^{2} q / \partial p_{0}^{2}\right)=\left(\partial^{2} h / \partial p_{0}^{2}\right)\left[1-\left(\partial p / \partial p_{0}\right)^{2}\right]=0 .
$$

QED. 
[1] J.-M. Mao and J. B. Delos, Phys. Rev. A 45, 1746 (1992).

[2] J. Main, G. Wiebusch, K. Welge, J. Shaw, and J. B. Delos, Phys. Rev. A 49, 847 (1994).

[3] Michael Courtney, Hong Jiao, Neal Spellmeyer, Daniel Kleppner, J. Gao, and J. B. Delos, Phys. Rev. Lett. 74, 1538 (1995).

[4] J. Gao and J. B. Delos, Phys. Rev. A 56, 356 (1997).

[5] A. D. Peters, C. Jaffé, J. Gao, and J. B. Delos, Phys. Rev. A 56, 345 (1997).

[6] J.-M. Mao, K. A. Rapelje, S. J. Blodgett-Ford, J. B. Delos, A. König, and H. Rinneberg, Phys. Rev. A 48, 2117 (1993).

[7] J. Gao and J. B. Delos, Phys. Rev. A 49, 869 (1994).

[8] G. Raithel, M. Fauth, and H. Walther, Phys. Rev. A 47, 419 (1993).

[9] Period-doubling bifurcations of periodic orbits are generic in one-parameter theory, and they are often called "pitchfork" bifurcations. We are discussing here pitchfork bifurcations that do not involve a doubling of the return time. Those are nongeneric.

[10] K. R. Meyer, Trans. Am. Math. Soc. 149, 95 (1970); 154, 273 (1971). See also K. R. Meyer, in Multiparameter Bifurcation Theory, Contemporary Mathematics Series Vol. 56, edited by M. Golubitsky and J. Guckenheimer (American Mathematical Society, Providence, RI, 1986), p. 373; K. R. Meyer and G. R. Hall, Introduction to Hamiltonian Dynamical Systems and the
N-Body Problem, Applied Mathematical Series Vol. 90 (Springer, New York, 1992).

[11] P. Schmelcher and L. S. Cederbaum, Phys. Rev. A 47, 2634 (1993) show some consequences of nuclear motions. If the electron "escapes" from the proton, then both electron and proton undergo cyclotron motion and $\vec{F} \times \vec{B}$ drift. After one proton cyclotron time, they come together again. We believe that this phenomenon is not relevant to the interpretation of the present experiments.

[12] Eugen Flöthmann and Karl H. Welge, Phys. Rev. A 54, 1884 (1996).

[13] S. Freund, R. Ubert, E. Flöthmann, K. Welge, D. M. Wang, and J. B. Delos (unpublished); D. M. Wang, Ph.D. thesis, College of William and Mary, 2000.

[14] A different SOS was suggested by Jaffé et al., but it is not convenient for the study of closed orbits [Charles Jaffé, David Farrelly, and T. Uzer, Phys. Rev. A 60, 3833 (1999)].

[15] One must be cautious about too closely associating $T_{u}$ and $T_{v}$ with $u$ and $v$ oscillations.

[16] T. Poston and I. Stewart, Catastrophe Theory and Its Applications (Pitman Publishers, London, 1978); R. Gilmore, Catastrophe Theory for Scientists and Engineers (WileyInterscience, New York, 1981). 